\title{
Identifying and anticipating tipping points in lake ecosystems
}

Peter G. Langdon', J.A. Dearing', J.G. Dyke' and R. Wang ${ }^{2}$

\begin{abstract}
Tipping points are large and potentially irreversible changes in a system. They have been extensively studied in lake ecosystems. This article describes how we can identify past and predict future tipping points and their impacts for lake management.
\end{abstract}

Lake ecosystems are changing rapidly under growing anthropogenic pressure, with many moving from one functioning state to another across poorly understood thresholds. These changes can be termed regime shifts, which we define as any substantial reorganization of a complex system with prolonged consequences. The cusp between states can be represented as a tipping point or critical transition, where a small perturbation may be sufficient to move the system from one state to another.

These abrupt, discontinuous changes feature internal processes and feedback loops which reinforce the new state, as in a fold bifurcation (Fig. 1). Consequently, being able to warn of an approaching tipping point before it is reached may allow for policies to be put in place in time.

Lake systems can be complex. But if we assume that the system can occupy more than one stable state, for example oligotrophic "clear water" or eutrophic "turbid water", then we can formulate simple mathematical models of how different drivers, such as nutrient loading or water-level change, cause the system to transition from one state to another. Such an approach is well established and early warning signals (EWS) or impending tipping points have been formulated for a range of natural and social systems (Scheffer 2009). Much of the original evidence and theory for tipping points and EWS has come from analyses and models of lake ecosystems, but more recently there has been a focus on the analysis of lake sediments (e.g. Wang et al. 2012).

As a lake ecosystem approaches a tipping point, it loses resilience. Models and laboratory experiments suggest that in this condition an ecosystem takes more time to recover from perturbations, a phenomenon known as critical slowing down (CSD). CSD is more likely a source of EWS in systems where the external impacts are relatively small. Where the impacts are large, the lake may flip temporarily in and out of an alternate state, before ultimately settling in the alternative state
- a phenomenon known as flickering. In lake ecosystems where external drivers constitute high levels of noise, flickering is a more likely source of EWS than CSD (Scheffer et al. 2012).

While the formulation of EWS is mathematically straightforward, there are a number of challenges in observing them in a real world context. For example, EWS require analysis of the rates of change of certain variables. This requires measurements taken over short intervals from long and highly resolved datasets that are sometimes not available. Furthermore, some studies have shown that regime shifts can arrive without warning (Hastings and Wysham 2010) and that EWS can arise as false positives in any model regardless of critical transitions (Kéfi et al. 2013). Additionally, many mathematical models used to define complex dynamics are low-dimensional simplifications of reality, which risk omitting the very complexity that characterizes the real-world systems Research priorities include observing or reconstructing EWS signals in time series from real-world lake systems so that relevant theories may be tested and the underlying mechanisms explored.

\section{Empirical examples}

Previous research shows that over the past 150 years many lakes have experienced regime shifts in their ecosystems through the effects of atmospheric pollution (acid rain from coal and oil fired power stations) or farming in the drainage basins (nutrient runoff from fertilizer applications) on water quality, and while some have since recovered to their former conditions many have not (Battarbee et al. 2014). While many

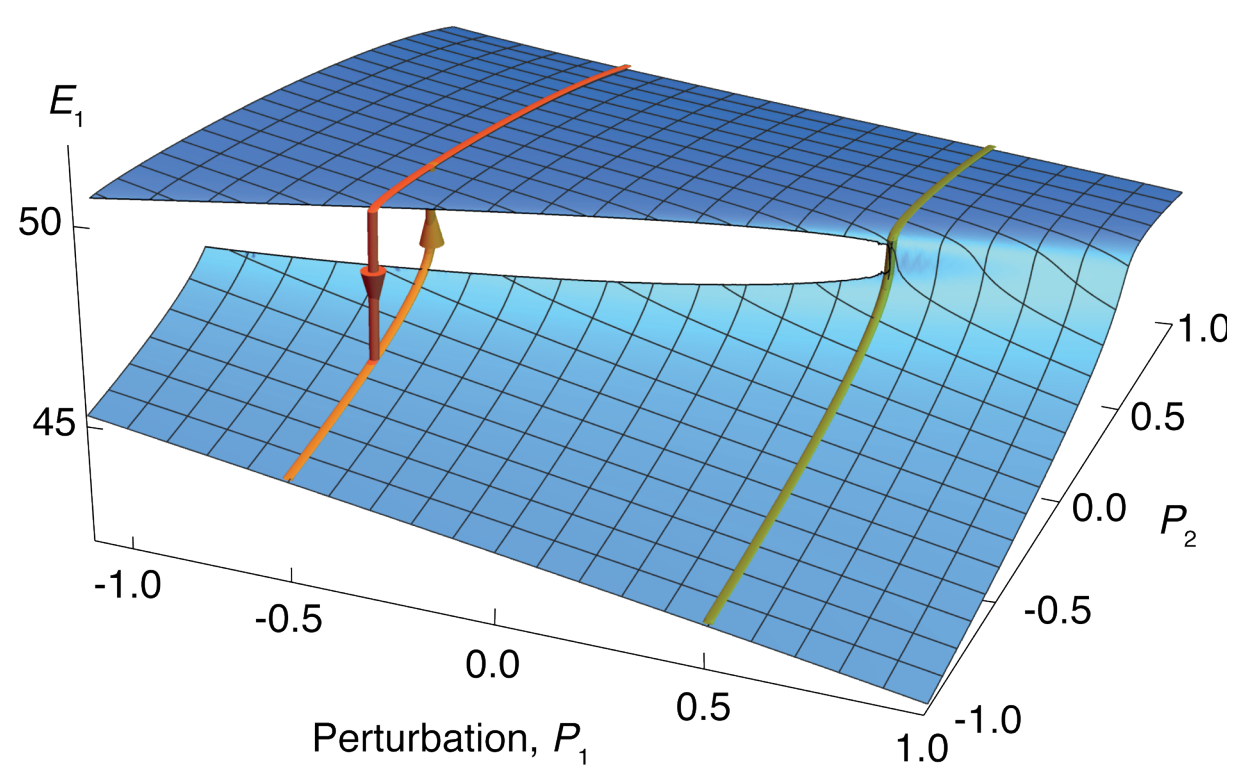

Figure 1: A simple model of a bi-stable system (Weaver and Dyke 2016). The diagram shows how the magnitude of perturbations (P1 and P2) acting on an environmental variable (E1) can lead to smooth, easy to reverse changes in the value of E1 (yellow line) or abrupt critical transitions with hysteresis loops (orange and red lines). 
studies have been undertaken on lake ecosystems in an attempt to identify transitions in state, relatively few have focused on detailed assessments of changes in resilience prior to transition, or attempted to identify EWS prior to a tipping point. Indeed, identifying characteristics of a tipping point, as opposed to more gradual shifts, remains a challenge for lake ecosystem research.

Shallow lake eutrophication typically results in a transition from benthic to pelagic community dominance that may or may not result in an overall shift in algal production. The drivers may be either top-down, such as significant changes in fish populations, or bottom-up through enhanced nutrient loading, or a combination of both. Evidence from temperate studies suggest that changes from clear water plant-dominated systems to turbid, eutrophic systems take 10-100s of years (Sayer et al. 2010). These changes could be interpreted as flickering between states, as the resilience drops and positive feedback loops strengthen. The final stage of settling into the alternative state would be an abrupt transition but further research is required to test these ideas.

Deeper lakes that stratify can also react to change through top-down or bottom-up processes. Some modeling work on instrumental and paleolimnological data has suggested that deep stratified systems do show nonlinear transitions between alternate attractors (Seekell et al. 2013). Other experiments on test lakes (with controls) in Michigan have manipulated the food web over a four-year period by steadily increasing the top predators to the ecosystem to turn the lake from turbid to clear water. All directly measured variables on the manipulated lake showed changes in variability (EWS) a year prior to the completion of the transition (Carpenter et al. 2011; Seekell et al. 2012). The tipping point appears to happen much faster than in shallow lakes, likely due to the speed and magnitude of the drivers (annual vs multi-decadal timescales). Can these effects be upscaled to a whole ecosystem? Batt et al. (2013) compared ecosystem productivity against measured drivers for the same manipulated and control lakes. They found no changes in variance, a typical EWS identified in modeling experiments, but they did define a "first day of alarm" that signifies an up-and-coming tipping point.

Other work on deeper lakes has focused on how increased nutrient loading can enhance internal nutrient recycling in a positive feedback cycle through hypolimnetic oxygen depletion. At Erhai Lake, China, diatom data showed a significant tipping point in 2001 using both equal and non-equal increment sediment samples. Monitored water quality and algal data before and after 2001 allowed reconstruction of the two stable states and the hysteresis effect predicted by bifurcation theory (Fig. 2). Time series analyses of sediment data suggested the presence of EWS in the
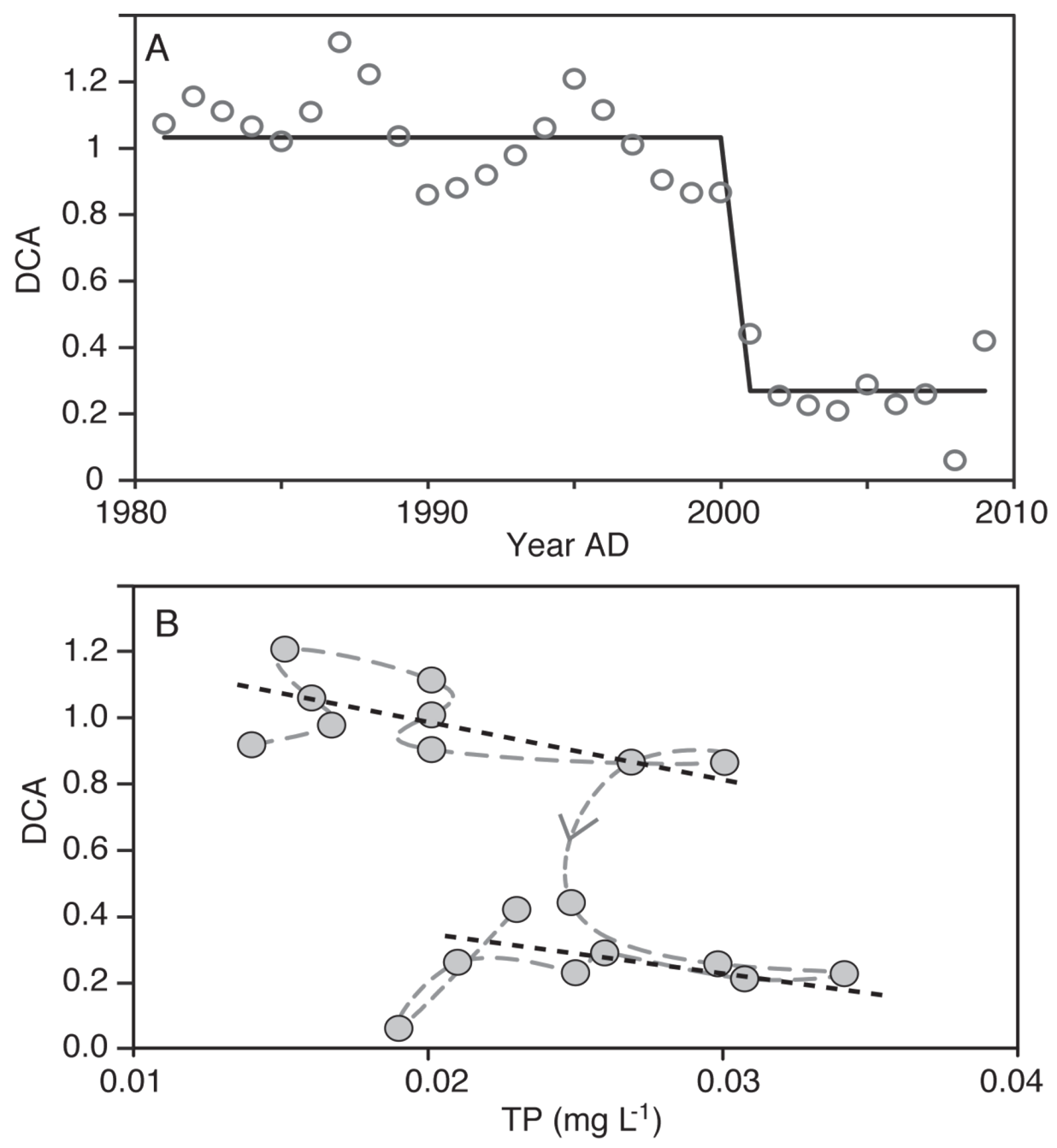

Figure 2: Regime shift, tipping point and alternative stable states at Erhai lake, Yunnan, China (after Wang et al. 2012). (A) Student's t-test on sequential analyses of diatom DCA data from deep water sediments which indicates a clear tipping point in 2001 from mesotrophic to hypereutrophic states. (B) Phase-space plot of instrumental measurements of total phosphorus (TP) from 1992-2009 plotted against changes in diatom state response (DCA axis 1 scores). The plot shows the two alternative states, the upper state (1992-2001) and lower state (2001-2009) occurred across TP values $0.02-0.03 \mathrm{mgl}^{-1}$, which is equivalent to $50 \%$ of the whole TP scale: this finding is strong evidence for alternative states and hysteresis.

form of increased variance a few decades before the transition (Wang et al. 2012; Doncaster et al., in press).

\section{Future challenges}

We do not yet understand why tipping points occur in some lakes and not others, nor are we able to identify EWS with confidence. There is much scope for more empirical data collection and analyses targeted at the detection of tipping points and EWS in lake ecosystems. More data will improve our modeling approaches and advance theoretical developments. Paleolimnological data offer great potential in these respects especially where time-series of sub-decadal ecological processes can be resolved at equal increments. Significant advances in identifying and anticipating tipping points in lakes and other systems are not only possible but eagerly awaited by the wide scientific community.

\section{AFFILIATIONS}

${ }^{1}$ Geography \& Environment, University of Southampton, UK

${ }^{2}$ Nanjing Institute of Geography and Limnology, Chinese Academy of Sciences, Nanjing, China

\section{CONTACT}

Peter G. Langdon: P.G.Langdon@soton.ac.uk REFERENCES

Batt RD et al. (2013) PNAS 110: 17398-17403

Battarbee RW et al. (2014) Ecol Indic 37: 365-380

Carpenter SR et al. (2011) Science 332: 1079-1982

Doncaster CP et al. (in press) Ecology, doi: 10.1002/ ecy. 1558

Hastings A, Wysham D (2010) Ecol Lett 13: 464-472 Kéfi S et al. (2013) Oikos 122: 641-648

Sayer CD et al. (2010) Freshwater Biol 55: 500-513 Scheffer M (2009) Critical Transitions in Nature and Society. Princeton University Press, 400 pp Scheffer M et al. (2012) Science 338: 344-348 Seekell DA et al. (2012) Ecosystems 15: 741-747 Seekell DA et al. (2013) Theor Ecol 6: 385-394 Wang R et al. (2012) Nature 492: 419-422

Weaver IS, Dyke JG (2016) Earth Syst Dyn Discuss doi:10.5194/esdd-6-2507-2015 